\title{
Differences in anthropometric and laboratory data, related to weight loss, in patients with heart failure and various tumors: is weight loss unique?
}

\author{
Tomislav Letilović1*, Stjepan Kranjčević1, Damir Kozmar ${ }^{1}$, Darko Počanić', \\ Ivica Premužić Meštrović', Helena Jerkićc ${ }^{1}$ Radovan Vrhovac ${ }^{2}$ \\ ${ }^{1}$ Merkur University Hospital, Zagreb, Croatia \\ ${ }^{2}$ University of Zagreb School of Medicine, University Hospital Centre Zagreb, Zagreb, Croatia
}

Objectives: Body weight loss is an important feature of heart failure (HF) and tumors. It is related to both reduced survival and adverse reactions to therapy in both of these conditions. The mechanisms of body weight loss in patients with HF and tumors are considered to be similar. Yet, studies comparing those two populations are generally lacking. The aim of this study was to compare anthropometric and laboratory data, related to weight loss, between the patients with chronic HF and the patients with different tumors as well as the control population.

Patients and Methods: The study was conducted at the Department of Internal Medicine of University Hospital Merkur in Zagreb. Data were collected from May 2011 until May 2012 in consecutive patients that were eligible for entry. We gathered data for 143 patients and 20 controls. In the patient group there were 45 patients with HF, 55 with solid hematological tumors and 43 with solid tumors of various sites.
Results: Patients with HF had lower levels of C-reactive protein (CRP) and albumin compared to controls. Anthropometric measurements revealed lower body mass index (BMI), muscle strength, mid-arm circumference and waist circumference in patients with HF compared to controls. Measurements of biceps, triceps, subscapular and suprailiac skinfolds were also lower in HF group. Compared to solid tumor group HF patients had lower levels of CRP and higher levels of hemoglobin. Solid tumor patients had lower values of BMI and subscapular skinfold thickness as well as higher muscle strength compared to HF group. Finally, compared to patients with solid hematological tumors, the HF group had lower levels of albumin, lower BMI, lower muscle strength as well as lower mid-arm circumference.

Conclusion: Our results show expected differences in anthropometric and laboratory features, related to weight loss, in patients with heart failure compared to control population. On the other hand, observed differences in HF group

Table 1. Differences in anthropometric and laboratory variables, related to weight loss, between heart failure group and controls. Values are given as a mean \pm standard deviation.

\begin{tabular}{|l|c|c|c|}
\hline & Heart failure & Controls & p-value \\
\hline Body weight $-\mathrm{kg}$ & $75.9 \pm 15.4$ & $84.4 \pm 16.7$ & $0.04^{*}$ \\
BMI $-\mathrm{kg} / \mathrm{m}^{2}$ & $26.5 \pm 4.8$ & $28.4 \pm 8.0$ & $0.04^{*}$ \\
Grip strength $-\mathrm{kg}$ & $17.2 \pm 9.7$ & $26.8 \pm 8.6$ & $0.0005^{*}$ \\
Mid-arm circumference $-\mathrm{cm}$ & $27.9 \pm 3.7$ & $30.4 \pm 3.4$ & $0.009^{*}$ \\
Waist circumference $-\mathrm{cm}$ & $94.0 \pm 11.2$ & $100.0 \pm 14.7$ & $0.03^{*}$ \\
Hip circumference $-\mathrm{cm}$ & $100.1 \pm 8.8$ & $100.4 \pm 10.1$ & $\mathrm{NS}$ \\
Biceps skinfold $-\mathrm{cm}$ & $0.75 \pm 0.44$ & $1.0 \pm 0.4$ & $0.008^{*}$ \\
Triceps skinfold $-\mathrm{cm}$ & $1.23 \pm 0.50$ & $1.48 \pm 0.45$ & $0.04^{*}$ \\
Subscapular skinfold $-\mathrm{cm}$ & $1.43 \pm 0.48$ & $1.87 \pm 0.5$ & $0.002^{*}$ \\
Suprailiac skinfold $-\mathrm{cm}$ & $1.28 \pm 0.52$ & $1.56 \pm 0.51$ & $0.04^{*}$ \\
CRP $-\mathrm{mg} / \mathrm{L}$ & $10.0 \pm 11.6$ & $4.3 \pm 5.5$ & $0.01^{*}$ \\
Hemoglobin $-\mathrm{g} / \mathrm{L}$ & $132.8 \pm 22.3$ & $136.8 \pm 13.8$ & $\mathrm{NS}^{*}$ \\
Albumin $-\mathrm{g} / \mathrm{L}$ & $37.9 \pm 4.5$ & $43.5 \pm 4.3$ & $0.001^{*}$ \\
\hline
\end{tabular}

${ }^{*} p<0.05$ - Mann - Whitney $U$ test. Abbreviations: $N S=$ not significant, $\mathrm{cm}=$ centimeters, $\mathrm{kg}=$ kilograms, $m=$ meters, $m g=$ milligrams, $g=$ grams, $L=$ liters. 
Table 2. Differences in anthropometric and laboratory variables, related to weight loss, between heart failure group and solid tumor group. Values are given as a mean \pm standard deviation.

\begin{tabular}{|lccc|}
\hline & Heart failure & Solid tumors & p-value \\
\hline Body weight $-\mathrm{kg}$ & $75.9 \pm 15.4$ & $73.4 \pm 16.9$ & $\mathrm{NS}$ \\
BMI $-\mathrm{kg} / \mathrm{m}^{2}$ & $26.5 \pm 4.8$ & $24.4 \pm 4.7$ & $0.04^{*}$ \\
Grip strength $-\mathrm{kg}$ & $17.2 \pm 9.7$ & $24.7 \pm 9.6$ & $0.008^{*}$ \\
Mid-arm circumference $-\mathrm{cm}$ & $27.9 \pm 3.7$ & $28.4 \pm 4.2$ & $\mathrm{NS}$ \\
Waist circumference $-\mathrm{cm}$ & $94.0 \pm 11.2$ & $94.8 \pm 12.6$ & $\mathrm{NS}$ \\
Hip circumference $-\mathrm{cm}$ & $100.1 \pm 8.8$ & $99.0 \pm 10.0$ & $\mathrm{NS}$ \\
Biceps skinfold $-\mathrm{cm}$ & $0.75 \pm 0.44$ & $0.66 \pm 0.29$ & $\mathrm{NS}$ \\
Triceps skinfold $-\mathrm{cm}$ & $1.23 \pm 0.50$ & $1.11 \pm 0.46$ & $\mathrm{NS}$ \\
Subscapular skinfold $-\mathrm{cm}$ & $1.43 \pm 0.48$ & $1.24 \pm 0.54$ & $0.04^{*}$ \\
Suprailiac skinfold $-\mathrm{cm}$ & $1.28 \pm 0.52$ & $1.18 \pm 0.60$ & $\mathrm{NS}$ \\
CRP $-\mathrm{mg} / \mathrm{L}$ & $10.0 \pm 11.6$ & $37.6 \pm 43.5$ & $0.0005^{*}$ \\
Hemoglobin $-\mathrm{g} / \mathrm{L}$ & $132.8 \pm 22.3$ & $122.8 \pm 18.5$ & $0.02^{*}$ \\
Albumin $-\mathrm{g} / \mathrm{L}$ & $37.9 \pm 4.5$ & $35.4 \pm 6.8$ & $\mathrm{NS}$ \\
\hline
\end{tabular}

${ }^{*} p<0.05$ - Mann - Whitney U test. Abbreviations: $N S=$ not significant, $\mathrm{cm}=$ centimeters, $\mathrm{kg}=$ kilograms, $m=$ meters, $m g=$ milligrams, $g=$ grams, $L=$ liters.

Table 3. Differences in anthropometric and laboratory variables, related to weight loss, between heart failure group and solid hematological tumors group. Values are given as a mean \pm standard deviation.

\begin{tabular}{|lccc|}
\hline Body weight $-\mathrm{kg}$ & Heart failure & $\begin{array}{c}\text { Solid hemato- } \\
\text { logical tumors }\end{array}$ & p-value \\
\hline BMI $-\mathrm{kg} / \mathrm{m}^{2}$ & $75.9 \pm 15.4$ & $80.7 \pm 18.0$ & $\mathrm{NS}$ \\
Grip strength $-\mathrm{kg}$ & $26.5 \pm 4.8$ & $27.5 \pm 5.5$ & $\mathrm{NS}$ \\
Mid-arm circumference $-\mathrm{cm}$ & $17.2 \pm 9.7$ & $26.3 \pm 10.2$ & $0.0001^{*}$ \\
Waist circumference $-\mathrm{cm}$ & $27.9 \pm 3.7$ & $31.1 \pm 4.1$ & $0.0004^{*}$ \\
Hip circumference $-\mathrm{cm}$ & $94.0 \pm 11.2$ & $96.3 \pm 14.9$ & $\mathrm{NS}$ \\
Biceps skinfold $-\mathrm{cm}$ & $100.1 \pm 8.8$ & $102.9 \pm 9.7$ & $\mathrm{NS}$ \\
Triceps skinfold $-\mathrm{cm}$ & $0.75 \pm 0.44$ & $0.84 \pm 0.35$ & $\mathrm{NS}$ \\
Subscapular skinfold $-\mathrm{cm}$ & $1.23 \pm 0.50$ & $1.35 \pm 0.45$ & $\mathrm{NS}$ \\
Suprailiac skinfold $-\mathrm{cm}$ & $1.43 \pm 0.48$ & $1.50 \pm 0.59$ & $\mathrm{NS}$ \\
CRP - mg/L & $1.28 \pm 0.52$ & $1.49 \pm 0.59$ & $\mathrm{NS}$ \\
Hemoglobin $-\mathrm{g} / \mathrm{L}$ & $10.0 \pm 11.6$ & $15.9 \pm 36.1$ & $\mathrm{NS}$ \\
Albumin $-\mathrm{g} / \mathrm{L}$ & $132.8 \pm 22.3$ & $127.1 \pm 22.6$ & $\mathrm{NS}$ \\
\hline
\end{tabular}

${ }^{*} p<0.05$ - Mann - Whitney $U$ test. Abbreviations: $N S=$ not significant, $\mathrm{cm}=$ centimeters, $\mathrm{kg}=$ kilograms, $m=$ meters, $m g=$ milligrams, $g=$ grams, $L=$ liters.

compared to patients with various tumors could imply different pathophysiological mechanisms of weight loss between those groups. Such data were not previously published and could serve as a cornerstone for studies with larger numbers of patients and deeper pathophysiological insight.

KEYWORDS: weght loss, heart failure, tumor.

CITATION: Cardiol Croat. 2013;8(9):291-292.
Received: $29^{\text {th }}$ Jun 2013

*Address for correspondence: Klinička bolnica Merkur, Zajčeva 19, HR-10000 Zagreb, Croatia.

$+385-1-2431390$

E-mail: letilovic@yahoo.com

\section{Literature}

1. Evans WJ, Morley JE, Argiles J, Bales C, Baracos V, Guttridge D et al. Cachexia: a new definition. Clin Nutr. 2008;28:793-9.

2. Morley JE, Thomas DR, Wilson MMG. Cachexia: pathophysiology and clinical relevance. Am J Clin Nutr. 2006;83:735-43.

3. Lainscak M, Filippatos GS, Gheorghiade M, Fonarow GC, Anker SD. Cachexia: common, deadly, with an urgent need for precise definition and therapies. Am J Cardiol. 2008;101:8E-10E 